\title{
A Review on Image Quality Improvement using Denoise Filtering Techniques
}

\author{
Pranali Turkhade ${ }^{1}$, Umesh Hore ${ }^{2}$ \\ P.G. Student, Electronics and Telecommunication Department, P. R. Patil College, Amravati, India ${ }^{1}$ \\ Professor, Electronics and Telecommunication Department, P. R. Patil College, Amravati, India ${ }^{2}$
}

\begin{abstract}
Image improvement is a processing on an image to make it more appropriate for certain applications. The Image denoising is the manipulation of the image data to produce a visually high quality image. The aim of image enhancement is to improve the visual appearance of an image or to provide a better transform representation for future image processes. At present there are a variety of methods to remove noise from digital images. Filter techniques are mainly used for de-noising, smoothness and sharpening of images. In this paper we are designed a new modified decision based unsymmetric and Symmetric trimmed median filter for removal of these different noises by using median filter, Adaptive median filter, and Decision based Adaptive median filter. Most of the previously known techniques are applicable for the denoising of images corrupted with less noise density. Here a new decision based technique has been presented which shows better performances than those already being used and the new proposed algorithm will definitely protect the image from noise and distortion. In this paper we are presented different denoise filtering techniques for image quality improvement and this concept is applied to the different images and compares their performance parameters such as MSE, PSNR, correlation etc.
\end{abstract}

Keywords: impulse noise, non linear filters, MSE, PSNR, correlation.

\section{INTRODUCTION}

Image quality improvement is related with the field of image processing. The digital image contains important information in the form of object and text that can be used for various purposes like object recognition, face recognition and text recognition. In most of the applications, under pre-processing phase image denoising techniques are involved to process image such that its visual quality can be improved. After the denoising, the improved quality image can be used for further processing. The aim of denoising technique is to remove unwanted signals from the image while saving important information. The noise in image is a disturbance that distorts the information and decreases the quality of the image. The noise is introduced in the image due to various reasons such as the imaging sensor may be affected by environmental conditions or due to insufficient Light levels and sensor temperature may introduce the noise in the image and another reason is interference in the transmission channel may also corrupt the image.

One of the most important areas of image restoration is that cleaning an image occurring by noise. In image processing field the goal of reducing noise is to eliminate noisy pixels Corruption of images .The noise is usually divided into Gaussian noise, the balanced noise and the impulse noise. One of the most common noise types which corrupt images during transmission is impulse noise also known as salt \& pepper. Salt and pepper noise not only corrupts true information of the image, but also seriously affects the visual effects of the image. Therefore, the reduction of impulse noises has important significance to image processing and computer vision analysis. In this type of noise, a pixel gets the minimum or maximum value that can take in a dynamic range of available values. A large number of algorithms have been proposed to remove this noise while still trying to preserve image details. For an image corrupted by noises, we can use linear or nonlinear filter methods to reduce noises. In the case of linear filtering, the noise reduction algorithm is applied for all pixels of the image linearly without knowing about noisy pixel and non-noisy pixel.

\section{LITERATURE REVIEW}

In the field of digital image processing filter plays an important role in the image de-noising process. It is a technique for modifying or enhancing an image. There are different papers in which many methods have been proposed by using multiple images by different authors are as follows.

One of the important implementation of Median Filtered Image Quality Enhancement and Anti-Forensics via Variational Deconvolution by Wei Fan, Kai Wang, Franc ois Cayre, and Zhang Xiong [1] have been proposed an image variational deconvolution framework for both quality enhancement and antiforensics of median filtered images. This method can serve as a median filtered image quality enhancement technique, whose efficacy is validated by experiments conducted on median filtered images which have been previously "salt \& pepper" noised. The proposed method outperforms the state-of-theart median filtering anti-forensics, with a better forensic undetectability against existing detectors as well as a higher visual quality of the processed image.

[2] Comparative Analysis of Median Filter and Adaptive Filter for Impulse Noise. The basic idea behind this analysis is the maximization of the similarities between pixels in a predefined filtering window. The comparison 
introduced to this median filter and adaptive filter lies in the establishment of parameters of the similarity function. The results show that the adaptive filter outperforms most of the basic algorithms for the reduction of impulsive noise in grey scale images.

Denoising of salt-and-pepper noise corrupted image using modified directional-weighted-median filter by Ching-Ta Lu, Tzu-Chun Chou [3] have been proposed many denoising algorithms to recover a noise corrupted image. The experimental results show that the proposed approach cannot only efficiently suppress high-density impulse noise, but also can well preserve the detailed information of an image.

An Improved Median Filtering Algorithm for Image Noise Reduction The algorithm uses the correlation of the image to process the features of the filtering mask over the image [4]. It can adaptively resize the mask according to noise levels of the mask. The statistical histogram is also introduced in the searching process of the median value. Experimental results show that the algorithm reduces the noise and retains the details of the image. The complexity of the algorithm is decreased to $\mathrm{O}(\mathrm{N})$, and the performance of noise reduction has effectively improved.

Another important implementation of Prof.R.Gayathri1, Dr.R.S.Sabeenian [5] have been presented A Survey on Image Denoising Algorithms (IDA), In which Several denoising procedures are proposed to preserve the image quality in textured images by removing the noise encountered .In this paper the effective noise removal techniques are discussed for various types of images and the suggestions for improving the interpretability or the perception of information in the image are listed.

A New Adaptive Weight Algorithm for Salt and Pepper Noise Removal [6] developed A new adaptive weight algorithm for the removal of salt and pepper noise. It consists of two major steps, first to detect noise pixels according to the correlations between image pixels, then use different methods based on the various noise levels. Experiments show the proposed algorithm has advantages over regularizing methods in terms of both edge preservation and noise removal, even for heavily contaminated image with noise level as high as $90 \%$, it still can get a significant performance.

\section{III.PROPOSED WORK}

Digital image quality improvement is a field of engineering that studies methods to recover an original scene from degraded observations. Often, the captured image may not be of good quality because of factors such as noise, poor brightness, contrast, blur, or artefacts. Figure shows the block diagram for image quality improvement using different filtering algorithms.

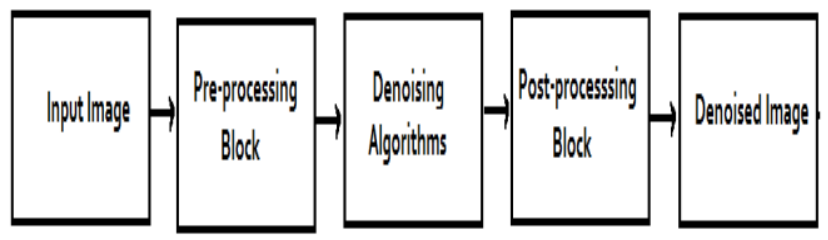

Fig 1: Block diagram of proposed work
The basic block diagram of analysis of image quality improvement consists of three major stages: Preprocessing, Denoising Algorithms, Post processing block.

\section{A. Input Image}

In the Digital Image Processing field, removing the noise from the image is the difficult issue. An image is unfortunately corrupted by various factors. The distortions of images by noise are common during its processing and transmission. These noisy effects decrease the performance of visual analysis. Gaussian noise (White noise), Salt \& Pepper noise and Speckle noise are the types of noises which are generally found in Images, and also denoising them with the help of some efficient technique is of main task. Noise when get added to image destroy the details of it. So in order to preserve the real image, noise should get removed from it. And for the purpose of enhancement the contrast of the image should be improved.

\section{B. Pre-processing block}

In this block different processes are done on the input image such as first convert the $3 \mathrm{D}$ visual information into $2 \mathrm{D}$ digital form. After that improve the image quality by enhancement, restoration and using different denoise filtering techniques. Several techniques have been proposed over the years for image filtering. Linear filtering techniques have been the methods of choice for many years for their mathematical simplicity and existence theory for their design and implementation nonlinear approaches have been found to be more effective for this purpose. Filters having good edge and image detail preservation properties are highly suitable for image filtering and enhancement. New algorithms and techniques, which can take advantage of the increase in computing power and can handle more realistic assumptions, are needed. Thus, the development of nonlinear filtering techniques such as median filter, adaptive median filter, decision based adaptive median filter, and our proposed method i.e. modified unsymmetric trimmed median filter which perform equally well under wide variety of applications, is of great importance.

\section{Denoising Algorithms}

1. Proposed algorithm:

Modified Unsymmetric Trimmed Mean Filter (MUTMF)

This MUTMF is our proposed algorithm when the Decision Based Algorithm fails to act for a processing pixel. Following figure shows the structure of MUTMF. The algorithm for MUTMF works as follows:

Step 1: A 2D 3x3 window is selected with the processing pixel at its centre.

Step 2: All the elements are transferred into a 1D array. Step 3: All ' 0 ' and '255' are eliminated from the 1Darray. Step 4: If the length of the $1 \mathrm{D}$ array is equal to 0 . The window size is increased ( $3 \times 3$ to $5 \times 5,5 \times 5$ to $7 \times 7$ and so on). Steps 2-4 are repeated again in case of increase in window size.

Step 5: The processing pixel Pxy is assigned a new value which is the mean of the 1D array.

Step 6: Move to the next pixel and repeat steps 1-5 for every pixel in the entire image. 
The output of this filter gives us the final pixel values after restoration.

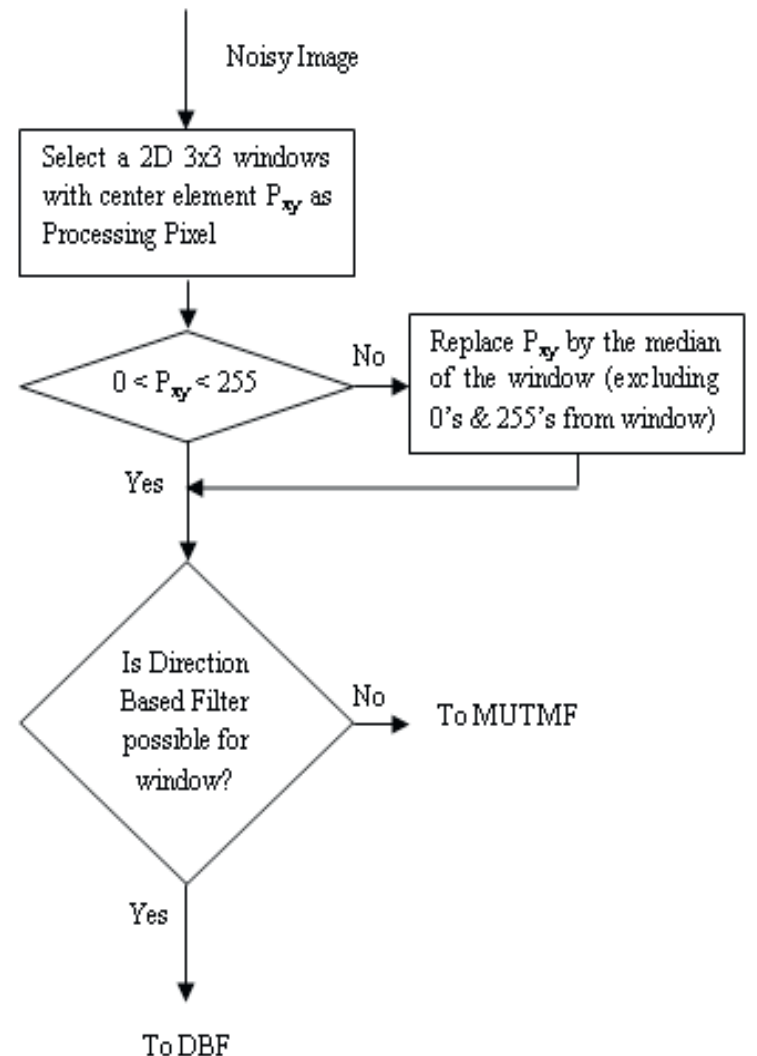

\begin{tabular}{c|c|c|c|}
\multicolumn{1}{c}{} & \multicolumn{1}{c}{$y-1$} & \multicolumn{1}{c}{$y$} & $y+1$ \\
\cline { 2 - 4 }$x-1$ & $a$ & $b$ & $c$ \\
\cline { 2 - 4 }$x+1$ & $d$ & $e$ & $f$ \\
\cline { 2 - 4 } & $g$ & $h$ & $i$ \\
\cline { 2 - 4 } & \multicolumn{3}{|c|}{ Window: 3x3 Mask }
\end{tabular}

Fig 2: structure of proposed work

\section{Post Processing Block:}

Most of the previously known techniques are applicable for the denoising of images corrupted with less noise density. Here a new decision based technique has been presented which shows better performances than those already being used and the new proposed algorithm will definitely protect the image from noise and distortion. In this paper we have analysed different denoise filtering techniques for image quality improvement and this concept is applied to the different images and they are compared with one another. The comparison between denoised images is taken in terms of performance parameters such as mean square error (MSE), peak signal to noise ratio (PSNR), correlation, etc.

\section{E. Denoised Image}

By using different filtering techniques we will get improved quality of image .depending on the noise density we will apply different algorithms to image and analysed the parameters of the denoised image.

\section{IV.CONCLSION}

Filters are used best for removing noise from the images. In this paper, a new algorithm has been proposed that increases the efficiency of the removal of salt and pepper noise. Results of this algorithm exhibit better performance in comparison with MF, AMF, DBA and MDBA in terms of higher PSNR, MSE and correlation. The proposed filter also shows consistent and stable performance for noise densities varying from $10 \%-90 \%$. The proposed filter shows better image enhancement factor for salt and pepper noise of density more than $70 \%$. As a future work the proposed algorithm can be further improved by implementation of de-blurring techniques in neural networks or fuzzy logic outputs and also to remove other types of noise such as speckle noise, random noise, Rayleigh noise, Gaussian noise, etc.

\section{REFERENCES}

[1] Wei Fan, Kai Wang, Franc, ois Cayre, and Zhang Xiong, "Median Filtered Image Quality Enhancement and Anti-Forensics via Variational Deconvolution", TIFS.2015.2398362, IEEE Transactions on Information Forensics and Security, 2015

[2] Rachna Mehta, Dr. Navneet Kumar Aggarwal, "Comparative Analysis of Median Filter and Adaptive Filter for Impulse Noise", International Journal of Computer Applications (0975 - 8887) National Conference on Recent advances in Wireless Communication and Artificial Intelligence (RAWCAI-2014)

[3] Ching-Ta Lu, Tzu-Chun Chou, "Denoising of salt-and-pepper noise corrupted image using modified directional-weighted-median filter", 33th Pattern Recognition Letters, 2012

[4] Youlian Zhu, Cheng Huang, "An Improved Median Filtering Algorithm for Image Noise Reduction”, 2012 International Conference on Solid State Devices and Materials Science.

[5] Prof.R.Gayathri1, Dr.R.S.Sabeenian, "A Survey on Image Denoising Algorithms (IDA)"International Journal of Advanced Research in Electrical, Electronics and Instrumentation Engineering Vol. 1, Issue 5, November 2012

[6] Chen Cong-ping, Wang Jian, Qin Wu, Dong Xiao-gang, "A New Adaptive Weight Algorithm for Salt and Pepper Noise Removal"'

[7] Gang Cao Yao Zhao Rongrong Ni Lifang Yu Huawei Tian, "Forensic detection of median filtering in digital images" Beijing Natural Science Foundation (No. 4073038).IEEE 2010

[8] Angalaparameswari Rajasekaran1, Senthilkumar, "Image Denoising Using Median Filter with Edge Detection Using Canny Operator", International Journal of Science and Research (IJSR) ISSN (Online): 2319-7064

[9] Mr. Rohit Verma Dr. Jahid Ali, “A Comparative Study of Various Types of Image Noise and Efficient Noise Removal Techniques" International Journal of Advanced Research in Computer Science and Software Engineering Volume 3, Issue 10, October 2013 ISSN: 2277 128X

[10] Chih-Yuan Lien, Chien-Chuan Huang, Pei-Yin Chen, Member, and Yi-Fan Lin, "An Efficient Denoising Architecture for Removal of Impulse Noise in Images and its VLSI architecture for the removal of random-valued impulse noise"

[11] Gaihua Wang, DehuaLi, WeiminPan, ZhaoxiangZang, "a modified switching median filter for noise reduction in colour images that are corrupted with impulse (salt and pepper) noise"

[12] Zhiyong Zuo, Tianxu Zhang, Jing Hu, Gang Zhou, "A new method for removing impulse noise based on noise space characteristic."

[13] Priyanka Kamboj and Versha Rani“"A brief study of various noise model and filtering techniques" IGRCS Volume 4, No. 4, April 2013 ISSN $2229371 \mathrm{X}$

[14] Suman Shrestha, "Image denoising using new adaptive based median filter" Signal \& Image Processing : An International Journal (SIPIJ) Vol.5, No.4, August 2014

[15] Roman Garnett, Timothy Huegerich, Charles Chui, "A Universal Noise Removal Algorithm with an Impulse Detector" IEEE 2010.

[16] K.M. Sharavana Raju, Mohammad Shahnawaz Nasir, T. Meera Devi, "Filtering Techniques to reduce Speckle Noise and Image Quality Enhancement methods on Satellite Images" IOSR Journal of Computer Engineering (IOSR-JCE) e-ISSN: 2278-0661, pISSN: 2278-8727Volume 15, Issue 4 (Nov. - Dec. 2013). 